\title{
La mecánica cuántica y la necesidad de incluirla en los planes de estudio
}

\section{Quantum mechanics and the need to include it in study plans}

\author{
Jeisson Martin Calvo \\ Alexander Martin Calvo ${ }^{2}$ \\ DOI: 10.29151/reit.n1a2
}

PProfesor Titular, Universidad Distrital Francisco José de Caldas. Profesor Asociado, Facultad de Ciencia y Tecnología Universidad Pedagógica Nacional. Colombia;

jfmartinc@pedagogica.edu.co, ORCID ID: https://orcid.org/0000-0002-2390-9677

${ }^{2}$ Profesional de Autoevaluación y Currículo, Unimonserrate. Colombia;

jamartin@unimonserrate.edu.co, ORCID ID: https://orcid.org/0000-0001-9718-9185 


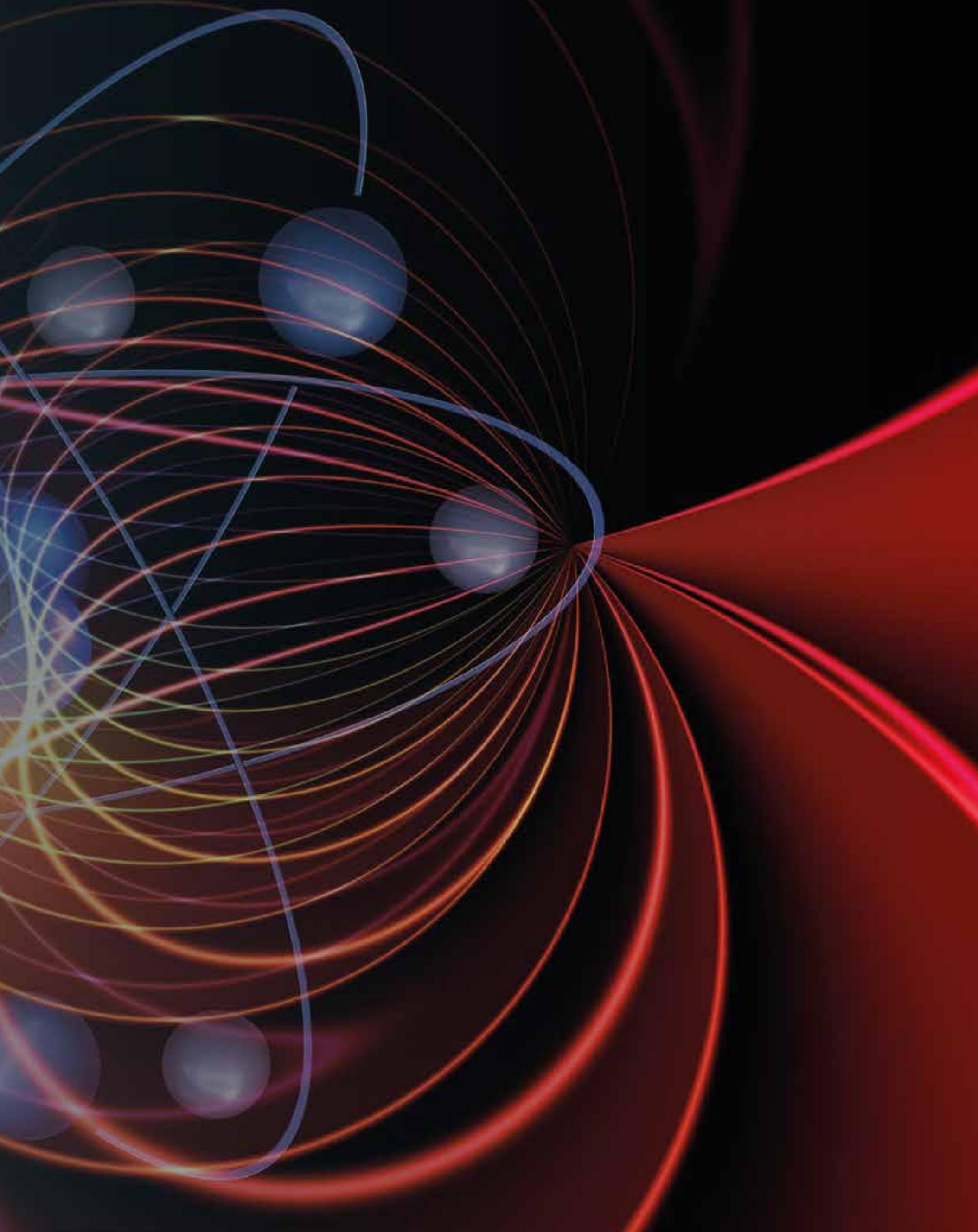




\section{Resumen}

En este trabajo se expresan las ideas que se generaron a partir de la elección del tema de mecánica cuántica (MC) y la reflexión sobre la necesidad de incluirla en los planes de estudio. Mediante el análisis de información, se planteó un fundamento teórico que resulto adecuado en términos epistemológicos con el fin de indagar de forma critica la inclusión de tópicos de mecánica cuántica en los diseños curriculares. Los resultados obtenidos se presentan desde los aspectos generales de la MC, hasta el aprendizaje y su enseñanza tomando como referente lo que se realiza en torno a este tema de investigación a nivel local e internacional. Donde se resalta la importancia de implementar en Colombia propuestas de investigación en enseñanza de las ciencias y la ingeniería que incluyan la aplicación de tópicos de física moderna.

Palabras clave: Diseño curricular; Enseñanza de la física; Mecánica cuántica; Física moderna. 


\begin{abstract}
In this paper we express the ideas that were generated from the choice of the topic of quantum mechanics (CM) and the reflection on the need to include it in the curricula. Through the analysis of information, a theoretical foundation was proposed that was adequate in epistemological terms in order to critically investigate the inclusion of quantum mechanics topics in the curricular designs. The results obtained are presented from the general aspects of $\mathrm{CM}$, to learning and teaching, taking as a reference what is done around this research topic at a local and international level. The importance of implementing research proposals in science and engineering education in Colombia that include the application of modern physics topics is highlighted.
\end{abstract}

Keywords: Curriculum Design; Physics Teaching; Quantum Mechanics; Modern Physics. 


\section{Introducción}

En este manuscrito de reflexión, se realiza un análisis de las visiones epistemológicas relacionadas con la mecánica cuántica MC. Del mismo modo, se hace una síntesis sobre los aspectos generales de la MC con el fin de indagar sobre algunas interpretaciones. Así mismo, se discute el problema de la medición en MC, la paradoja del gato de Schrödinger y se describe la paradoja EPR que sirvió como pauta para producir diversas interpretaciones de la teoría cuántica. Se presenta, el análisis de hechos experimentales desde la visión de Feynman de la experiencia de la doble rendija. También, se discute sobre las revoluciones científicas y el aprendizaje de las ciencias. Partiendo de este análisis, se plantean las siguientes preguntas de conocimiento: ¿Cuáles son los fundamentos teóricos generales de la MC?, ¿Qué implica la enseñanza y el aprendizaje de la mecánica cuántica? ¿Porque enseñar nociones de física cuántica en la escuela? ¿Se debe incluir la mecánica cuántica en el diseño curricular y los planes de estudio en Colombia?

En una aproximación a la revisión de la literatura para acotar el tema de la enseñanza de la MC se tomó como referencia a Fanaro (2009), Greca y Moreira (2001), Ostermann y Moreira (2000) y Paulo (2006). En la búsqueda de la información se tuvieron en cuenta aspectos referentes a la enseñanza desde una visión histórica y epistemológica de la física cuántica; que se pueden encontrar en Gil y Solbes (1993) y Castañeda (2005). Sobre las ideas de los investigadores entorno a los contenidos curriculares de la mecánica cuántica se encontró en Vicario \& Venier (2010) un referente sobre el debate de la enseñanza de la física en América latina donde se expone la realidad de la enseñanza de la mecánica cuántica en países como Brasil, Argentina y Colombia, planteando un debate sobre la inclusión de nociones cuánticas en los planes de estudio Por último, se revisaron las propuestas didácticas sobre la enseñanza de nociones cuánticas en cursos de educación media y universitarios haciendo referencia a los trabajos desarrollados por Paulo y Moreira (2004) y otras visiones donde se utiliza el enfoque de caminos múltiples de Feynman como el trabajo realizado por Ogborn (2003) del Instituto de Educación de la Universidad de Londres donde plantean un currículo de física basado en nociones de física moderna desde la perspectiva cuántica de Feynman.

\section{Materiales y métodos}

Este manuscrito es de carácter reflexivo documental, para formalizar una aproximación teórica sobre la mecánica cuántica y abordar las preguntas de conocimiento planteadas con el fin de abordar de forma critica la necesidad de incluirla en los diseños curriculares, se realizaron las heurísticas de estudios tomados de bases de datos como EBSCO, Dialnet, Latindex, Redalyc, y scielo entre otras. Para obtener dichos trabajos se realizaron búsquedas de acuerdo a la temática planteada y aspectos como relevancia, índices de citación y palabras clave entre otros.

\section{Resultados y discusión}

Para la reflexión sobre la enseñanza de la mecánica cuántica, se planteó un fundamento teórico que hace referencia a aspectos generales de la $M C$, el problema de la medición en MC, la paradoja EPR, los hechos experimentales, ¿Qué involucra la enseñanza y el aprendizaje de la MC y la Enseñanza de la MC en Colombia que se presentan a continuación: 


\section{Aspectos generales de la MC}

La mecánica cuántica estudia el comportamiento de la materia y ha contribuido no solo al desarrollo del pensamiento científico, sino también a la forma de ver el mundo y de relacionarnos con éste. Su aplicación ha contribuido al desarrollo de instrumentos tecnológicos que facilitan la comunicación como satélites, teléfonos celulares, computadores cuánticos y dispositivos electrónicos, entre otros. La MC ha estado acompañada de análisis filosóficos y epistemológicos que marcaron precedentes esenciales en la comprensión de la naturaleza.

Debido al surgimiento de la relatividad y la MC la física se fraccionó en dos partes la macroscópica y la microscópica, admitiendo la teoría de la relatividad y la cuántica como la física moderna. La relatividad hizo replantear el aspecto epistemológico subjetivo de la física. Sin embargo, con el transcurso del tiempo la subjetividad continuó influyendo en el estudio de fenómenos naturales lo que implica que no se podía abandonar. Esto tiene que ver con que la probabilidad es subjetiva y está relacionada con el conocimiento que se tiene de las cosas, siendo esta categorizada como trascendental para las leyes físicas por la MC. La probabilidad, se puede considerar como un concepto peculiar ya que, para obtener un resultado detallado y verdadero, primero se crea un conocimiento impreciso de lo que es probable. Lo que parece ir en contra de la seguridad en donde el conocimiento es lo establecido y los hechos son los actos. Además, de manera habitual la probabilidad es considerada como lo opuesto a los hechos, en cuanto a que si algo es probable no debe tomarse como un hecho real.

La teoría cuántica plantea que los hechos que han sido observados son probabilidades, entonces el resultado de una observación definido como un hecho tiene valor científico pues da la posibilidad de que otro hecho ocurra. En comparación con la física clásica, la teoría cuántica enuncia que los resultados de las mediciones experimentales pueden establecer un conocimiento inexacto de lo observado y las probabilidades son en realidad lo objetivo. El indeterminismo de la física cuántica indica que los valores de las cantidades que pueden predecirse son incertidumbres, son valores que se pueden conocer con el pasar del tiempo. El principio de Heisenberg plantea que la posición de un electrón en un instante de tiempo sólo puede ser conocida con incertidumbres dependientes entre sí y la posición del electrón un segundo después de la observación tiene incertidumbre. De esta forma, no es posible conocer simultáneamente la posición y la velocidad. El problema de la incertidumbre en el conocimiento señalado por Heisenberg establece la relación entre teoría y realidad, afirma que en el proceso del desarrollo de conceptos científicos se presentan idealizaciones expresadas de forma axiomática que se producen de las experiencias, generando que se acoplen a esquemas matemáticos; esta idealización suscita la pérdida del contacto con la realidad, tal que: "los conceptos siguen ajustándose no muy bien a aquella realidad parcial que ha construido el objeto de investigación" (Heisenberg, 1963, p.131).

La MC afirma que lo que realmente se observa son probabilidades. Por lo tanto, se puede plantear que la síntesis del conocimiento teórico de la física moderna se relaciona con la observación de forma irreversible y esta relación resulta análoga al concepto de probabilidad. Esto permite que los conocimientos derivados de hechos experimentales se interpongan en el determinismo, lo cual genera el indeterminismo de la física moderna en términos de su contenido experimental. Se puede 
decir que el resultado de un proceso experimental en física es una afirmación, pero con la aceptación de la probabilidad la afirmación se limita a su grado.

Por otra parte, la MC cuestiona la validez del concepto de causalidad que establece la regla de la causa y el efecto. El concepto de causa se ha transformando en el tiempo ya que la percepción del hombre por los fenómenos de la naturaleza ha cambiado. Inicialmente, la causa hacía referencia a algo que antecedía una acción para explicar qué la provocó. Heisenberg (1985), mencionó que cuando algo sucede se supone que algo ocurrió previamente. El significado del concepto de causalidad se fue transformando hasta ser interpretado como una serie de eventos naturales determinados de tal forma que lo que sucede es suficiente para predecir el futuro. La física newtoniana está estructurada bajo este significado y si se conoce el estado inicial de un sistema en determinado tiempo, se puede determinar su futuro comportamiento. Partiendo de la causalidad, Laplace insinuó que, si en un instante se conoce la posición y el movimiento de átomos, sería viable calcular el destino del universo. Cuando se da una interpretación como la anterior a la causalidad se caería en determinismo causal. La física permaneció inclinada al determinismo hasta las investigaciones de Planck sobre la teoría de la radiación, donde se demostró que un átomo radiante no lanzaba su energía de forma continua sino discontinua. El suministro discontinuo de energía llevo a formular la hipótesis respecto a la emisión de radiación como un fenómeno estadístico en concordancia con la teoría atómica que posteriormente se reformuló haciendo uso de la estadística, dejando atrás el determinismo.

Por un lado, en física cuántica se tiene la incertidumbre planteada por Heisenberg y por otro lado la segunda formulación presentada por Bohr donde se presentó la discrepancia con la postura de Heisenberg por la no inclusión del dualismo entre ondas y partículas. Luego se estableció que ambas posturas eran equivalentes y que la incertidumbre es un caso particular de una dualidad conocida como la complementariedad entendida como: "diferentes imágenes intuitivas destinadas a describir los sistemas atómicos pueden ser todas perfectamente adecuadas a determinados experimentos, a pesar de que se excluyan mutuamente" (Heisenberg, 1985, p.35).

Un átomo es representado como un sistema que tiene un núcleo atómico en el centro y electrones que giran en torno a el núcleo, en concordancia con el modelo de Bohr. Sin embargo, en otros experimentos se representa el núcleo atómico envuelto por un sistema de ondas estacionarias de frecuencia que determinan la radiación del átomo. Cada representación del átomo es verídica si se usa en el análisis adecuado, pero son incongruentes unas con otras. Los experimentos que evidencian la naturaleza ondulatoria y corpuscular de la materia exigen la formulación de regularidades estadísticas. En los fenómenos macroscópicos, la estadística de la física cuántica no cumple un papel trascendental porque al aplicar las leyes a estos fenómenos se obtienen altas probabilidades, estableciendo comportamientos que son determinados.

\section{El problema de la medición en MC}

El conocimiento científico depende de la experimentación y los resultados preliminares son corregidos por otros con nuevas condiciones. El método experimental derivado del positivismo, exige que la física tenga un proceso definido de observación estableciendo el problema de la medida. 
En física clásica la medición no es un problema, pero en física cuántica sí. En la clásica el sistema actúa en el instrumento de medición modificándolo y obteniendo un valor de medida, pero el observador puede ignorar el aparato de medida realizando una inferencia sobre el valor del observable. Si esto sucede, se supone despreciable la acción del aparato sobre el sistema. Por el contrario, en la física cuántica es importante incluir un tipo de acción que va del instrumento de medida al sistema medido. En la teoría cuántica, los instrumentos de medición que se utilizan no son adecuados para observar un átomo sin perturbarlo. En MC se considera el proceso de medición como un problema, porque en este proceso no se le asocian valores puntuales a los observables. El cambio de un estado inicial del sistema antes de la medición al estado final donde al observable se le asocia un valor, crea un colapso de estado. Se genera el interrogante si es posible que el observador genere este colapso, para responder esto se han planteado paradojas entre estas el gato de Schrödinger.

La paradoja del gato de Schrödinger consiste en que, dentro de una caja cerrada donde no se puede observar, se ubica un gato vivo y un núcleo radiactivo. Al emitir la correspondiente radiación se activa un dispositivo que liberará un gas venenoso y producirá la muerte del gato. Si se desea saber el estado del gato en un futuro instante se debe plantear un estado combinado gato vivo y gato muerto. Esta paradoja expresa que en $\mathrm{MC}$ al observar un sistema todos los estados son posibles y que al hacer una medición el sistema cae en colapso. En esta paradoja se observaría el gato vivo o muerto, pero únicamente en el instante que se abre la caja para realizar la observación. Esta característica de la $\mathrm{MC}$ genera el rompimiento de la objetividad y el determinismo.

\section{La paradoja EPR}

Einstein, Podolsky y Rosen (EPR) plantearon una paradoja que generó un debate cuántico ya que este sirvió como pauta para producir interpretaciones de la teoría cuántica. Einstein planteó un estado corpuscular para las ondas electromagnéticas, luego de Broglie esbozó el estado ondulatorio de los corpúsculos; después se planteó la ecuación de Schrödinger obteniendo soluciones que conciernen a ondas, las cuales Born interpretó de forma probabilística. Posteriormente, Heisenberg propuso el principio de incertidumbre generando el indeterminismo en $\mathrm{MC}$.

Einstein indagó sobre la relación tiempo y energía del principio de incertidumbre. Con un experimento mental, intentaba probar que la MC era incompleta. El experimento que planteó consistía en un sistema cuántico representado por una caja llena de fotones, en este sistema cuántico se deja escapar un fotón por medio de un obturador en un tiempo corto, que cuenta con un reloj de precisión ubicado en la caja. El principio de incertidumbre no permite decretar la energía del fotón con precisión. Pero Einstein en contravía a este principio planteo que la energía, en este caso se podría determinar pesando la caja justo antes y después de que el fotón escapara. Partiendo de la diferencia en la masa de la caja se podría dar la energía del fotón. De esta manera, logró poner en duda el principio de incertidumbre que es considerado como fundamental en MC.

Por otra parte; Bohr utilizó la teoría de la relatividad para refutar la idea de Einstein. La relatividad plantea que un intervalo de tiempo medido por un reloj se desplaza influido por un campo gravitacional que se evidencia en el corrimiento al rojo. Bohr planteó que el reloj es desplazado al moverse la caja de fotones cumpliendo con la re- 
lación que el producto de la energía y el intervalo de tiempo es mayor igual a la constante de Planck poniendo a salvo la MC. En 1935 sale a la luz la paradoja EPR que partía de una postura filosófica realista y suponía que el formalismo de la teoría cuántica era completo y el sistema separable. La EPR demostró que la MC no es completa y se planteó que si la lógica clásica es válida el formalismo de la MC aceptaba el realismo y con ello la separación de los sistemas. Idea que la MC no contemplaba, dando paso a diferentes interpretaciones de la teoría de los cuantos.

\section{Hechos experimentales}

Feynman manifestó que la MC podía evidenciarse con el experimento de la doble rendija, Young (1802) propuso este experimento obteniendo como resultado el comportamiento ondulatorio de la luz. Según Martin (2014), en este diseño experimental Young hizo incidir un haz de luz sobre un diafragma circular y colocó dos agujeros en una cartulina, luego cambio los agujeros por rendijas mejorando la calidad de la señal luminosa, Young describió su experiencia sin expresar ningún desarrollo matemático ni la fuente que utilizó, lo que historiadores de la ciencia como Worral (1976), interpretan como un experimento mental.

Posteriormente, en 1905 se halla que la luz también se comporta como corpúsculos o fotones confirmando el carácter dual de la luz ondapartícula. De esta manera, si se desea estudiar el comportamiento corpuscular de la luz con el experimento de la doble rendija se disminuye la intensidad de la luz, si se realiza la experiencia tapando la rendija derecha lo que se observa es una línea iluminada, si luego se tapa la otra rendija los fotones pasan por la rendija derecha que está abierta y lo que se observa es que se forma una línea semejante a la anterior, pero corrida hacia la derecha porque los fotones pasan a través de la rendija derecha. Si ahora se dejan abiertas las dos rendijas los puntos que se ven iluminados en el caso de una de las rendijas se verán oscuros y viceversa.

Las explicaciones basadas en la física clásica no permiten la comprensión de este fenómeno que se da como resultado del comportamiento ondulatorio de las partículas y genera el fenómeno de interferencia. Lo que se esperaría obtener en un experimento con partículas es que al hacer el experimento con las dos rendijas se observe en la pantalla la suma de las partículas que pasan a través de la rendija derecha más las que pasan por la izquierda. Sin embargo, el resultado que se obtiene es distinto, lo que acontece es que el fotón pasa por las dos rendijas al mismo tiempo.

Feynman (1965), interpreta el efecto de interferencia que se origina afirmando que el electrón o el fotón hacen lo que quiere. Este experimento arroja un resultado sorprendente ya que si se quiere saber por dónde pasa cada fotón y se instalan detectores en cada rendija desaparece del patrón de difracción observándose el comportamiento corpuscular de la luz. En el caso de no observarlo, su comportamiento es de carácter ondulatorio. Es decir que la observación cambia la realidad.

\section{¿Qué involucra la enseñanza y el aprendizaje de la MC?}

La mecánica cuántica ha sido fundamental en el desarrollo de la humanidad ya que ha influido en campos de la actividad cognitiva como la filosofía y epistemología. La MC es la base para entender la estructura de la materia de orden microscópico y ha forzado el cambio de la interpretación clásica de fenómenos. La llegada de la MC planteó una transformación sobre la medida de magnitudes físicas de sistemas cuánticos; hecho que se formali- 
za con el principio de incertidumbre dejando atrás el determinismo de la física clásica y adoptando la probabilidad que suceda un evento.

En la actualidad la tecnología se desarrolla con base en la MC por ejemplo la computación cuántica, criptografía, seguridad informática, superconductividad y la nanotecnología. La aplicación de la MC es evidente en la electrónica de los aparatos que se utilizan habitualmente; siendo ésta fundamental en la comprensión del presente y del futuro de la sociedad. Es por esto que la cuántica se debe incluir en los diseños curriculares para que los estudiantes se apropien de un conocimiento científico, cultural y cotidiano que les permita comprender el mundo en el que viven, analicen el futuro y los cambios tecnológicos.

Un primer paso a seguir en la enseñanza de la MC es que se aborde a partir de fenómenos simples con el fin de conceptualizar. Así mismo, los estudiantes deben ir más allá de las experiencias sensoriales y acudir a modelos que contienen propiedades abstractas. Chinn y Brewer (1993), plantean que el estudio del cambio conceptual en el mundo científico es una herramienta para entender la construcción de conocimiento en la clase de ciencias. Una de las formas de lograr el cambio conceptual para indagar sobre el mundo microscópico es remitirse a la historia y filosofía de la MC. Basándose en la historia y epistemología de las ciencias se ha relacionado la manera en que los científicos se enfrentan al conocimiento desde la investigación y en como los estudiantes asimilan el conocimiento científico sobre los fenómenos de la naturaleza que se contrapone a sus creencias. Trabajos como los de Gil y Solbes (1993), mostraron la implementación en el aula en España desde esta visión, concluyendo que los estudiantes lograron diferenciar la concepción cuántica de la clásica. Castañeda (2005), propone reflexiones históricas y epistemológicas para tener en cuenta en la enseñanza y el aprendizaje de la física cuántica pero no realiza la aplicación.

La epistemología es una herramienta que permite el entendimiento e interpretación de las teorías científicas y el análisis filosófico. Si el contexto de aprendizaje no promueve un desarrollo conceptual estableciendo significados se originará un fracaso en la construcción de nuevos modelos mentales esenciales en el razonamiento y la comprensión de las ideas científicas en MC. Sin embargo, una indagación epistemológica facilitará la construcción de un nuevo modelo mental que reconozca el mundo a escala microscópica; siendo esta un instrumento en la enseñanza de la física, en este caso en la enseñanza de la MC.

Sin embargo, el paradigma del cambio conceptual fue abandonado y el marco teórico de la investigación sobre la enseñanza de la MC sugiere abordarla desde la teoría de campos conceptuales (TCC) de Vergnaud (1990) y los lineamientos didácticos desarrollados por Otero $(2008,2011)$ sin hacer énfasis en una visión estrictamente histórica del desarrollo de la MC y utilizando una matemática acorde a la formación de los estudiantes.

Las propuestas relacionadas con el enfoque de Feynman nombradas en Fanaro (2009), utilizan la técnica de Caminos Múltiples centradas en el comportamiento de la luz con fotones utilizando herramientas computacionales, Fanaro (2009) utiliza el enfoque de la integral de caminos como herramienta en el análisis del comportamiento cuántico de electrones desde la aceptación de su comportamiento corpuscular utilizando el experimento de la doble rendija con proyectiles muy pequeños hasta llegar a los electrones, indagando de esta forma sobre los principios básicos que plantea la mecánica cuántica como el principio de superposición de estados, el de incertidumbre y el de correspondencia contribuyendo a la inves- 
tigación en el área de la enseñanza de la mecánica cuántica.

\section{Enseñanza de la MC en Colombia}

En Jaramillo, Arroyave, Higuita, \& López (2012), se afirma que en Colombia durante el proceso de enseñanza de la física en la mayoría de programas de pregrado no se abordan teorías de la física moderna como la MC. Se considera aquí que el estudio del comportamiento cuántico transformaría la enseñanza de la física, que en Colombia se encuentra limitada a la enseñanza de la física clásica. En el ámbito nacional los trabajos encontrados fueron elaborados por investigadores de la Universidad Nacional de Colombia, la Universidad Pedagógica Nacional, la Universidad de Antioquia y la Universidad Distrital Francisco José de Caldas sobre la enseñanza de la MC en cursos universitarios. Algunos estudios se centraron en reflexiones netamente históricas y epistemológicas, mientras otros en aspectos disciplinares con desarrollos matemáticos complejos.

El trabajo realizado por Mendoza \& Rozo (2011), presenta una reflexión sobre el principio de superposición de estados partiendo del concepto de estado de la mecánica clásica y de la MC afirmando que a los estudiantes se les dificulta la comprensión de este principio. También plantean el uso de analogías desde un contexto clásico y cuántico para la conceptualización y establecen el principio de superposición desde la teoría clásica y la teoría cuántica, en contraparte con el uso de conceptos clásicos para la interpretación de nociones cuánticas se afirma que:
Los resultados de las investigaciones sobre las ideas de los estudiantes acerca de conceptos cuánticos parecen sugerir que los estudiantes investigados no logran formar modelos mentales que les permitan visualizar cuánticamente la fenomenología microscópica. 0 sea, los nuevos conceptos, necesarios para tal descripción, son entendidos a partir de núcleos derivados de la fenomenología y la visión clásica, de forma que los estudiantes no consiguen elaborar explicaciones o predicciones en concordancia con las científicamente aceptadas. (Greca \& Herscovitz, 2002. p.329).

En la revisión de la literatura respecto a la investigación sobre la enseñanza de la MC en Colombia se encontraron pocos referentes. Vale la pena rescatar el trabajo realizado por Moreno \& Guarín (2010), que desarrollan un estudio de la enseñanza de nociones básicas de MC como la dualidad de la materia y el principio de superposición de estados. Los autores aproximan un grupo de estudiantes a concepciones alternativas de los conceptos antes mencionados desde la teoría cuántica. En el trabajo se tuvo en cuenta las dificultades al presentar las nociones cuánticas y diseñaron una estrategia didáctica basada en el uso de analogías, discusiones, montajes experimentales sobre oscilaciones armónicas y el uso de videos sobre el experimento de la doble rendija. La estrategia se aplicó en tres momentos. El primero sobre la idea de pequeño; el segundo sobre la dualidad de la materia y el tercero sobre el principio de superposición de estados. Los resultados les permitieron suponer que es posible implementar temas de mecánica cuántica en la escuela media colombiana. Sin embargo, investigadores de la enseñanza de la MC como Greca y Moreira (2000) y Fischler \& Lichtfeldt (1992) recomiendan explicar los fenómenos observados a partir de la interpretación estadística y evitar las descripciones duales en 
contraposición a lo que se desarrolla en el segundo y tercer momento del trabajo.

Habitualmente los estudiantes creen que la física está ligada a conocimientos que conciernen a problemas específicos que se plantean en los textos; y no al vínculo de concepciones y principios adaptables a fenómenos. Según Martin (2014), esto sucede debido a que el aprendizaje pasivo se ha convertido en un proceso habitual en la enseñanza de las ciencias; sometiendo el proceso de la enseñanza a aspectos teóricos y descontextualizados que se han convertido en acciones usuales, estableciendo condiciones en el proceso de enseñanza donde el docente y los libros son las autoridades. Para Sanmarti (2002), algunas de las razones del fracaso en la enseñanza de las ciencias apuntan entre otros factores a la falta de formación de los docentes en las disciplinas, al planteamiento curricular desactualizado de las asignaturas y al desconocimiento de estrategias metodológicas que les permitan desarrollar los temas haciendo uso de herramientas que resulten atrayentes para los estudiantes.

Partiendo de lo expuesto en eventos de divulgación científica como la escuela de Física y Matemáticas organizada por la Universidad de Los Andes, que ha abordado temas de Teorías cuánticas conformes y sus aplicaciones, Sistemas integrables cuánticos y las matemáticas del enredamiento, entre otros y del debate sobre la inclusión de la enseñanza de la física moderna en Latinoamérica esbozado a partir de los trabajos presentados en la X Conferencia Interamericana sobre Educación en física realizada en Medellín (Colombia). Donde se plantearon los avances que se han logrado en la enseñanza de la mecánica cuántica en países como Brasil y Argentina. Se determino incluir dentro del diseño curricular y el plan de estudios de los programas de ingeniería de la Fundación
Universitaria Monserrate abordar en algunos espacios académicos tópicos de la física moderna, con el fin de obtener una claridad conceptual de la MC con los estudiantes de la Escuela de Ingenierías y tecnologías. Para Arriasecq (2008), estas reformas introducidas por varios países en los currículos han impuesto una actualización de los mismos, introduciendo temas que se encuadran en la física contemporánea.

En la actualidad no tiene sentido aferrarse a la creencia que la física moderna, particularmente la mecánica cuántica está restringida a la comunidad científica, sino que es posible hacerla parte de la cultura de una población mediante la aplicación de didácticas específicas de la enseñanza de la física como la estructura conceptual de referencia propuesta por (Fanaro, 2009) donde la integral de caminos múltiples de Feynman (1949) se usa con el fin de realizar un diseño didáctico para analizar el comportamiento cuántico de electrones utilizando la experiencia de la doble rendija. Haciendo evidente que la teoría cuántica no es algo difícil ni abstracto si el componente matemático es adaptado y no como se acostumbra ser presentada con una formulación canónica, temática que debe formar parte del conocimiento elemental que alimenta la cultura de una población.

Con el fin de abordar el problema de la enseñanza de la MC, se sugiere tomar como referencia los trabajos realizados por Fanaro, Arlego, \& Otero (2006); Arlego (2008); Fanaro, Arlego, \& Otero, (2006); Fanaro, Arlego, \& Otero (2007); Arlego (2008); Mendoza Cely \& Rozo Clavijo (2011); Vicario \& Venier (2010); Castañeda (2005); Fanaro (2009); Greca (2000); Ostermann \& Moreira, (2000);Simas (1999); Greca \& Moreira, (2004); Greca, Moreira, \& Herscovitz (2001) y Paulo (2006). 


\section{Conclusiones}

La baja asimilación de contenidos científicos genera el interés en promover cambios curriculares dirigidos a superar las dificultades respecto a la escasa apropiación de conceptos de la física moderna.

En la literatura sobre la enseñanza de la MC en Colombia se encontraron pocos referentes, en general la enseñanza de la física moderna en el país se encuentra restringida a conceptos clásicos y las temáticas que se refieren a la MC no se incluyen en los currículos. Las investigaciones que se encontraron hacen referencia al estudio de la enseñanza de la $\mathrm{MC}$ en carreras de pregrado y posgrados como física, química y algunas ingenierías unas desde una visión netamente histórica y filosófica y otras disciplinares con estricto desarrollo matemático. Es por esto que surge la necesidad de incluir tópicos de física moderna en los diseños curriculares y en los planes de estudio en educación secundaria y universitaria.

En las experiencias que se analizaron sobre la enseñanza de la MC en colombiana se identificó desde la óptica de investigadores de la enseñanza de la MC que tienen dificultades en términos del uso de analogías con la física clásica y la utilización de descripciones duales. Además del uso de matemática complicada en contraposición a la utilización de una descripción estadística.

\section{Agradecimientos}

A la Escuela de Tecnologías e Ingenierías de la Fundación Universitaria Unimonserrate, al Núcleo de Investigación en Ciencias para Ingeniería Sanitaria NICIS y al programa de Licenciatura en Ciencias naturales y educación ambiental de la Universidad Pedagógica Nacional.

\section{Referencias}

Arlego, M. (2008). Los fundamentos de la mecánica cuántica en la escuela secundaria. Revista Electrónica de Investigación en Educación en Ciencias, 59-66.

Arriasecq, I. (2008). La enseñanza y el aprendizaje de la Teoría Especial de la Relatividad en el nivel medio/polymodal (tesis doctoral). Universidad de Burgos. España.

Castañeda Gallego, L. F. (2005). Algunas reflexiones históricas y epistemológicas a tener en cuenta en la enseñanza y el aprendizaje de la física cuántica (tesis especializacion en docencia universitaria). Universidad de Caldas (Colombia).

Chinn, C., \& Brewer, W. F. (1993). The role of anomalous data in knowledge acquisition: A theoretical framework and implications for science instruction. Review of Educational Research, 63, 1-49.

Fanaro, M. (2009). La Enseñanza de la Mecánica Cuántica en la Escuela Media. (Tesis Doctoral). Universidad de Burgos. Burgos.

Fanaro, M., Arlego, M., \& Otero, M. R. (2006). Los caminos múltiples de Feynman y la mecánica cuántica en la escuela media. Actas del 8 Simposio de Investigación en Educación en Física Asociación de Profesores de Física de la Argentina (APFA), 161-169.

Fanaro, M., Arlego, M., \& Otero, M. R. (2007). El método de caminos múltiples de Feynman para enseñar los conceptos fundamentales de la Mecánica Quántica en la escuela secundaria secundaria. Caderno Catarinense de Ensino de Física, 233-260.

Feynman, R. (1999 p.28). El placer de descubrir. Drakonos.

Feynman, R. (Vol III 1965). Lectures on Physics. 
Feynman, R. (1987). Física, Volumen III, Mecánica cuántica. Addison-Wesley Iberoamericana S.A.

Fischler, \& Lichtfeldt. (1992). Modern Phisycs and students' conceptions. International Journal of Science education, 181-190.

Greca, I. (2000). Construindo Significados Em Mecânica Quântica: Resultados De Uma Proposta Didática Aplicada a Estudantes De Física Geral (Tesis Doctoral).

Greca, I. M., \& Moreira, M. A. (2001). Uma Proposta para o Ensino de Mecânica Quântica. Revista Brasileira de Ensino de Física, vol. 23, no. 4, 444-457.

Greca, I. M., Moreira, M. A., \& Herscovitz, V. (Dezembro, 2001). Uma Proposta para o Ensino de Mecânica Quântica. Revista Brasileira de Ensino de Física, vol. 23, no. 4, 444-457.

Greca, I, \& Herscovitz, V. (2002). Construyendo significados en mecanica cuantica: Fundamentacion y resultados de una propuesta innovadora para su introduccion en el nivel Universitario. Revista enseñanza de las Ciencias, Vol20, 329.

Greca, I., \& Moreira, M. A. (2004). Obstáculos representacionales mentales en el aprendizaje de conceptos cuánticos Instituto de Física. UFRGS, Brasil.

Heisenberg, W. (1963). El papel de la física moderna. Revista Universitas, 131.

Heisenberg, W. (1985). La imagen de la naturaleza en la física actual. Barcelona: Ediciones Orbis.

Jaramillo Zapata, J. E., Arroyave Echavarría, J. E., Higuita Giraldo, J. D., \& López Ríos, S. Y. (2012). Una aproximación al despertar de la enseñanza de la física. Revista EDUCyT, 18.

Martin Calvo, J. F. (2014). Aprendizaje activo aplicado a la ensenanza del fenomeno optico de interferencia de la luz en el marco del proyecto ondas de colciencias en la Isla de San Andres. Universidad Nacional de Colombia. Bogotá.

Mendoza Cely, D. M., \& Rozo Clavijo, M. (2011). el principio de superposición de estados, revista científica. Volumen Extra. Año 2011, 61-65.

Moreira, M. A., \& Greca, I. (2000). Em Atas do III Encontro Internacional sobre Aprendizagem Significativa. Introduçao a Mecânica Quântica: seria o caso de evitar a aprendizajem significativa (subordinada) Peniche, Portugal.

Moreno, H., \& Guarin, E. (2010). Nociones cuánticas en la escuela secundaria: Un estudio de caso. Lat. Am. J. Phys. Educ. Vol. 4, No. 3, Sept. 2010, 669-676.

Ostermann, F., \& Moreira, M. A. (2000). Física contemporánea en la escuela secundaria: una experiencia en el aula involucrando formación de profesores. Investigación didactica, 391-404.

Otero, M. R. (2008). Actas del V Semana de Investigación Programa Internacional De Doctorado En Enseñanza De Las Ciencias UBU/ UFRGS. Enseñanza de las Ciencias: Aspectos Didácticos, Cognitivos y Afectivos. Porto Alegre. Ostermann, F., \& Moreira, M. A. (200). Física contemporánea en la escuela secundaria: una experiencia en el aula involucrando formación de profesores. Investigación didáctica, 391-404.

Paulo, J. C. (2006). A Aprendizagem Significativa Crítica de Conceitos de da Mecânica Quântica Segundo a Interpretação de Copenhagen e o Problema da Diversidade de Propostas de Inserção da Física Moderna e Contemporânea no Ensino Médio.Tesis Doctoral.

Pérez, R. (s.f.). La teoría de la Relatividad, la Mecánica Cuántica, La eternidad y otras Temá- 
ticas... Editorial de la Universidad Tecnológica Nacional.

Sanmarti, N. (2002). Necesidades de formación del profesorado en función de las finalidades de la enseñanza de las ciencias. Pensamiento Educativo Vol. 30, pp.35-60.

Simas Alvetti, M. A. (1999). Ensino de física moderna e contemporânea e a revista ciência hoje. Florianópolis, 1999. Dissertação (Mestrado em Educação) - Faculdade de Educação, Universidade Federal de Santa Catarina. Florianópolis.
Vicario, J., \& Venier, F. (2010). La enseñanza de la física moderna, en debate en Latino America. Revista Argentina de Enseñanza de la Ingeniería / Año 11 / № 20 / Julio / 2010.

Worral. (1976). Thomas young and the refutation of newtonian optics: a case-study in the interaction of philosophy of sicience and the history of science. Cambridge: university press.

Young. (1802). On the theory of light and colours, a Bakerian lecture. 